\title{
Lagrangian formalism of gravity in the Randall-Sundrum model
}

\author{
Petter Callin* and Finn Ravnda ${ }^{*}$ \\ Department of Physics, University of Oslo, N-0316 Oslo, Norway
}

(Dated: October 3, 2005)

\begin{abstract}
We derive the effective Lagrangian of the physical four-dimensional fields in the Randall-Sundrum (RS) model, and use this to calculate the Newtonian gravitational potential between two point sources on the brane. The effect of the radion is emphasized, and it is shown to disappear when the hidden brane is taken to infinity, i.e. when we only have one brane. As a preliminary, we derive the corresponding Lagrangian in the simpler geometry of a flat spacetime with one extra dimension compactified on a torus. We also study the gauge invariance of the theory, and note that the massless vector field that is present for the torus disappears in the RS model because of the orbifold symmetry.
\end{abstract}

PACS numbers: 04.50.+h, 04.62.+v, 11.10.Kk, 98.80.Jk

\section{INTRODUCTION}

In this paper we develop the effective four-dimensional Lagrangian of gravity in theories with extra dimensions, and in particular we study the fine-tuned RandallSundrum (RS) model 1, 2]. The derivation include both the RS2 model with a single brane 2], and also the more phenomenologically interesting RS1 model with two branes [1]. In the latter case, the four-dimensional Lagrangian includes a massless scalar field (radion), a result that is typical of theories with compact extra dimensions. The Lagrangian is then used to calculate the Newtonian gravitational potential between two static point sources on the visible brane.

Theories with extra dimensions, and in particular the braneworld model of Randall and Sundrum, have attracted a lot of attention recently [3, 4, 5, 6, 7, 8, 9, 10]. One central issue is whether effective four-dimensional gravity is restored at the scales currently probed by experiments, i.e. whether the gravitational potential $V(r) \approx-G m_{1} m_{2} / r$ at those scales. There seems to be three different ways of calculating the potential. One way is to calculate the component $h_{00}$ of the metric perturbation due to a matter source directly [3, 4, 5]. This method, however, usually leads to complications with brane-bending, i.e. the fact that the branes can no longer be considered straight when introducing a matter source on them. A different approach is to consider the gravitational potential as the result of two massive particles interacting through the exchange of a virtual graviton, and to simply use the wave equation of the graviton to determine its propagator and interaction with matter $6,77,8]$. With this approach the branes are no longer bent, because the gravitons are actually travelling through empty space. However, it can be difficult to fix the normalization of the different four-dimensional fields from their

\footnotetext{
*Electronic address: n.p.callin@fys.uio.no
}

†Electronic address: finn.ravndal@fys.uio.no wave equations alone, and this normalization is crucial in order to get the correct relative contribution from the radion as compared to the four-dimensional gravitons. The most reliable and consistent approach is therefore to derive the Lagrangian of the five-dimensional graviton, do a dimensional reduction to four dimensions, and then identify the physical four-dimensional fields by requiring that they have canonical Lagrangians [9]. We are going to use this approach throughout the paper.

In section III we derive the Lagrangians and propagators of both massless and massive gravitons in $D$ spacetime dimensions, so that this can be used as a reference to identify the physical graviton fields later on. In section III we study the simplest possible case with extra dimensions, that is flat space with one dimension compactified on a torus. The main features of the derivation will be the same as for the warped RS geometry, only with much simpler algebra. This serves as a training ground before moving on to the RS model in section IV Finally, we conclude in section $\nabla$

\section{THE LAGRANGIAN AND PROPAGATOR OF SPIN-2 FIELDS IN FLAT SPACE}

\section{A. Massless spin-2 fields}

With an arbitrary number $D$ of spacetime dimensions, the action of gravity is given by

$$
S=-\frac{1}{2} M^{D-2} \int d^{D} x \sqrt{g} R,
$$

where $M$ is the Planck mass in $D$ dimensions, and $R$ the scalar curvature. The Lagrangian of gravitons in flat space is obtained by considering the perturbation

$$
g_{\mu \nu}=\eta_{\mu \nu}+2 M^{(2-D) / 2} h_{\mu \nu}
$$

to the flat space metric $\eta_{\mu \nu}$. The factor $M^{(2-D) / 2}$ is chosen to give $h_{\mu \nu}$ the correct physical dimension $\operatorname{dim}[h]=$ $(D-2) / 2$, and the factor 2 gives the canonical normalization of the graviton field $h_{\mu \nu}$. Eq. (2) is then inserted 
into (11) and the result expanded to the second order in $h_{\mu \nu}$. After a straightforward calculation which involves several partial integrations, we find $S=\int d^{D} x \mathcal{L}_{h}$, where the graviton Lagrangian $\mathcal{L}_{h}$ is

$$
\mathcal{L}_{h}=-\frac{1}{2} h_{, \alpha} h^{, \alpha}+\frac{1}{2} h_{\alpha \beta, \nu} h^{\alpha \beta, \nu}+h^{\alpha \nu}{ }_{, \alpha} h_{, \nu}-h^{\alpha \nu}{ }_{, \alpha} h^{\beta}{ }_{\nu, \beta} .
$$

So far, everything we have done has been valid in an arbitrary gauge. However, in order to invert the quadratic operator and find the graviton propagator, we must fix the gauge to be used in the Lagrangian. The Lagrangian (3) is invariant under the infinitesimal coordinate transformation

$$
x^{\mu} \rightarrow \bar{x}^{\mu}=x^{\mu}+2 M^{(2-D) / 2} \xi^{\mu}(x),
$$

which transforms the graviton field as

$$
h_{\mu \nu} \rightarrow \bar{h}_{\mu \nu}=h_{\mu \nu}-\left(\partial_{\mu} \xi_{\nu}+\partial_{\nu} \xi_{\mu}\right) \text {. }
$$

The harmonic gauge where $\bar{h}^{\mu \nu}{ }_{, \nu}-\frac{1}{2} \bar{h}^{, \mu}=0$ is obtained by choosing $\square \xi^{\mu}=h^{\mu \nu}{ }_{, \nu}-\frac{1}{2} h^{, \mu}$. In this gauge, we can therefore add the gauge fixing term

$$
\mathcal{L}_{h g}=\frac{1}{\xi} C_{\mu} C^{\mu}, \quad C^{\mu}=h^{\mu \nu}{ }_{, \nu}-\frac{1}{2} h^{, \mu},
$$

to the Lagrangian. The action then becomes

$$
\begin{aligned}
S= & \int d^{D} x \frac{1}{2} h^{\mu \nu}\left[\left(1-\frac{1}{2 \xi}\right) \eta_{\mu \nu} \eta_{\alpha \beta} \partial^{2}\right. \\
& -\frac{1}{2}\left(\eta_{\mu \alpha} \eta_{\nu \beta}+\eta_{\mu \beta} \eta_{\nu \alpha}\right) \partial^{2} \\
& +\left(\frac{1}{\xi}-1\right)\left(\eta_{\mu \nu} \partial_{\alpha} \partial_{\beta}+\eta_{\alpha \beta} \partial_{\mu} \partial_{\nu}\right) \\
& +\frac{1}{2}\left(1-\frac{1}{\xi}\right)\left(\eta_{\mu \alpha} \partial_{\nu} \partial_{\beta}+\eta_{\mu \beta} \partial_{\nu} \partial_{\alpha}\right. \\
& \left.\left.+\eta_{\nu \alpha} \partial_{\mu} \partial_{\beta}+\eta_{\nu \beta} \partial_{\mu} \partial_{\alpha}\right)\right] h^{\alpha \beta} \\
\equiv & \int d^{D} x \frac{1}{2} h^{\mu \nu} \square_{\mu \nu \alpha \beta} h^{\alpha \beta} .
\end{aligned}
$$

The graviton propagator $D_{\alpha \beta \rho \sigma}\left(x, x^{\prime}\right)$ is defined as the Green's function inverse to the operator $\square_{\mu \nu \alpha \beta}$, i.e.

$$
\square_{\mu \nu \alpha \beta} D^{\alpha \beta \rho \sigma}\left(x, x^{\prime}\right)=\frac{1}{2}\left(\delta_{\mu}^{\rho} \delta_{\nu}^{\sigma}+\delta_{\mu}^{\sigma} \delta_{\nu}^{\rho}\right) \delta\left(x-x^{\prime}\right) .
$$

The solution can then be written as

$$
D_{\alpha \beta \rho \sigma}\left(x, x^{\prime}\right)=\int \frac{d^{D} k}{(2 \pi)^{D}} \frac{P_{\alpha \beta \rho \sigma}(k)}{k^{2}} e^{-i k \cdot\left(x-x^{\prime}\right)},
$$

where the polarization tensor $P_{\alpha \beta \rho \sigma}(k)$ is given by

$$
\begin{aligned}
& P_{\alpha \beta \rho \sigma}(k)=\frac{1}{2}\left(\eta_{\alpha \rho} \eta_{\beta \sigma}+\eta_{\alpha \sigma} \eta_{\beta \rho}\right)-\frac{1}{D-2} \eta_{\alpha \beta} \eta_{\rho \sigma} \\
& +\frac{(\xi-1)}{2 k^{2}}\left(\eta_{\alpha \rho} k_{\beta} k_{\sigma}+\eta_{\alpha \sigma} k_{\beta} k_{\rho}+\eta_{\beta \rho} k_{\alpha} k_{\sigma}+\eta_{\beta \sigma} k_{\alpha} k_{\rho}\right) .
\end{aligned}
$$

Thus, we see that the de Donder gauge where $\xi=1$ is a natural choice. The result (10) is in agreement with 11].

The interaction between gravitons and matter which is described by the Lagrangian $\mathcal{L}_{m}$, is obtained by including the action $S_{m}=\int d^{D} x \sqrt{g} \mathcal{L}_{m}$. Varying this action with respect to the metric, we then get $S_{\text {int }}=$ $\int d^{D} x \sqrt{g} \frac{1}{2} T_{\mu \nu} \delta g^{\mu \nu}$, and thus

$$
S_{\mathrm{int}}=-\int d^{D} x M^{(2-D) / 2} T^{\mu \nu} h_{\mu \nu},
$$

by using (2) to the lowest order in $h_{\mu \nu}$.

\section{B. Massive spin-2 fields}

The massless graviton in the previous section is given a mass by adding the Fierz-Pauli term $-\frac{1}{2} m^{2}\left(h^{\alpha \beta} h_{\alpha \beta}-h^{2}\right)$ to the Lagrangian (3). We thus have the Lagrangian of a massive spin-2 field

$$
\begin{aligned}
\mathcal{L}_{h}^{m}= & -\frac{1}{2} h_{, \alpha} h^{, \alpha}+\frac{1}{2} h_{\alpha \beta, \nu} h^{\alpha \beta, \nu}+h^{\alpha \nu}{ }_{, \alpha} h_{, \nu} \\
& -h^{\alpha \nu}{ }_{, \alpha} h^{\beta}{ }_{\nu, \beta}-\frac{1}{2} m^{2}\left(h^{\alpha \beta} h_{\alpha \beta}-h^{2}\right) .
\end{aligned}
$$

The resulting equation of motion for the free, massive graviton is

$$
\begin{aligned}
0= & \square_{\mu \nu \alpha \beta}^{m} h^{\alpha \beta}=\eta_{\mu \nu}\left(\partial^{2}+m^{2}\right) h-\left(\partial^{2}+m^{2}\right) h_{\mu \nu} \\
& -\eta_{\mu \nu} \partial_{\alpha} \partial_{\beta} h^{\alpha \beta}-\partial_{\mu} \partial_{\nu} h+\partial_{\mu} \partial^{\alpha} h_{\alpha \nu}+\partial_{\nu} \partial^{\alpha} h_{\alpha \mu}
\end{aligned}
$$

Taking the derivative and contracting this equation, we obtain the constraints

$$
\partial^{\mu} h_{\mu \nu}=0, \quad h=\eta^{\mu \nu} h_{\mu \nu}=0
$$

One thus obtains the simplified equation of motion

$$
-\square_{\mu \nu \alpha \beta}^{m} h^{\alpha \beta}=\left(\partial^{2}+m^{2}\right) h_{\mu \nu}=0,
$$

which is why the Fierz-Pauli term seems a natural way of giving the graviton a mass.

With this mass term included, there is no gauge freedom in the Lagrangian. The operator $\square_{\mu \nu \alpha \beta}^{m}$ can therefore be inverted directly, leading to the propagator

$$
D_{\alpha \beta \rho \sigma}^{m}\left(x, x^{\prime}\right)=\int \frac{d^{D} k}{(2 \pi)^{D}} \frac{P_{\alpha \beta \rho \sigma}^{m}(k)}{k^{2}-m^{2}} e^{-i k \cdot\left(x-x^{\prime}\right)},
$$

with the polarization tensor

$$
\begin{aligned}
& P_{\alpha \beta \rho \sigma}^{m}(k)=\frac{1}{2}\left(\eta_{\alpha \rho} \eta_{\beta \sigma}+\eta_{\alpha \sigma} \eta_{\beta \rho}\right)-\frac{1}{D-2} \eta_{\alpha \beta} \eta_{\rho \sigma} \\
& \quad-\frac{1}{2 m^{2}}\left(\eta_{\alpha \rho} k_{\beta} k_{\sigma}+\eta_{\alpha \sigma} k_{\beta} k_{\rho}+\eta_{\beta \rho} k_{\alpha} k_{\sigma}+\eta_{\beta \sigma} k_{\alpha} k_{\rho}\right) \\
& \quad+\frac{1}{(D-1)(D-2)}\left(\eta_{\alpha \beta}+\frac{D-2}{m^{2}} k_{\alpha} k_{\beta}\right)\left(\eta_{\rho \sigma}+\frac{D-2}{m^{2}} k_{\rho} k_{\sigma}\right) .
\end{aligned}
$$

This result was also found in [1] for the case $D=4$. 


\section{TORUS COMPACTIFICATION}

We first consider the case of flat $(4+1)$-dimensional space, where the extra dimension is compactified on a torus with circumference $L$. This allows us to get comfortable with the formalism before moving on to the more complicated RS model. It also allows us to do the whole calculation of the gravitational potential from a purely five-dimensional point of view, so that we can compare the results when doing a four-dimensional decomposition of the fields. Corrections to the gravitational potential with torus compactification was also studied in [12, 13.

\section{A. Five-dimensional point of view}

From a five-dimensional point of view, the graviton is massless, and the polarization tensor in the propagator is given by (10) with $D=5$. The interaction vertex is $M^{-3 / 2} T_{\mu \nu}$. For a point particle with mass $m$ at rest at the point $(\boldsymbol{x}, y), y$ being the coordinate in the fifth dimension, the energy momentum tensor is

$$
T_{\mu \nu}(\boldsymbol{x}, y)=m u_{\mu} u_{\nu} \delta(\boldsymbol{x}) \delta(y)=m \delta_{\mu}^{0} \delta_{\nu}^{0} \delta(\boldsymbol{x}) \delta(y),
$$

and thus

$$
T_{\mu \nu}(\boldsymbol{k}, p)=m \delta_{\mu}^{0} \delta_{\nu}^{0}
$$

where $p$ is the momentum in the fifth dimension. The gravitational potential between two point particles with masses $m_{1}$ and $m_{2}$ is therefore

$$
\begin{aligned}
V(\boldsymbol{k}, p) & =\lim _{k^{0} \rightarrow 0} \\
& =\lim _{k^{0} \rightarrow 0}\left[\frac{m_{1} m_{2}}{M^{3}} \frac{P_{0000}(k)}{k_{0}^{2}-\boldsymbol{k}^{2}-p^{2}}\right] \\
& =-\frac{2 m_{1} m_{2}}{3 M^{3}} \frac{1}{\boldsymbol{k}^{2}+p^{2}} .
\end{aligned}
$$

In particular, we note that the gauge dependent term in (10) disappears, because only the 0000-component of the polarization tensor contributes, and $k^{0} \rightarrow 0$. When Fourier inverting (20), the finite length of the fifth dimension means that the momentum $p$ is quantized as $p_{n}=2 \pi n / L, n=0, \pm 1, \pm 2, \ldots$, with the result

$$
\begin{aligned}
V(r, y) & =\int \frac{d^{3} k}{(2 \pi)^{3}} \frac{1}{L} \sum_{p} V(\boldsymbol{k}, p) e^{i \boldsymbol{k} \cdot \boldsymbol{x}} e^{i p y} \\
& =-\frac{m_{1} m_{2}}{6 \pi M^{3} L r} \sum_{p} e^{-|p| r+i p y} \\
& =-\frac{m_{1} m_{2}}{6 \pi M^{3} L r} \frac{\sinh \frac{2 \pi r}{L}}{\cosh \frac{2 \pi r}{L}-\cos \frac{2 \pi y}{L}}
\end{aligned}
$$

where $r=|\boldsymbol{x}|$. This is the same result as obtained in [13].

Of particular importance is the limit of large distances, where the fifth dimension disappears from the potential, and we are left with the four-dimensional result

$$
\lim _{r \rightarrow \infty} V(r)=-\frac{m_{1} m_{2}}{6 \pi M^{3} L r} \equiv-\frac{m_{1} m_{2}}{8 \pi M_{\mathrm{Pl}}^{2} r}=-\frac{G m_{1} m_{2}}{r}
$$

where $M_{\mathrm{Pl}}$ is the effective four-dimensional Planck mass, and $G$ the gravitational constant. We therefore obtain

$$
M_{\mathrm{Pl}}^{2}=\frac{3}{4} M^{3} L .
$$

The factor $3 / 4$ may be a bit surprising. In the next section we will see that it is the combination of a fourdimensional graviton and scalar field that both contribute to the long range gravitational force that gives this factor. Of course, it is possible to define the fourdimensional Planck mass by only considering the spin-2 fields that contribute to the long range force, obtaining the common $M_{\mathrm{Pl}}^{2}=M^{3} L$. In that case, however, the potential will no longer have the usual form $V(r)=-G m_{1} m_{2} / r$, but also an additional contribution due to the scalar field. It therefore seems natural to also include the scalar mode in the definition of the four-dimensional Planck mass.

As a consistency check, we can also consider the limit of (21) for short distances, i.e. $r, y \rightarrow 0$ :

$$
\lim _{r, y \rightarrow 0} V(r, y)=-\frac{m_{1} m_{2}}{6 \pi^{2} M^{3}\left(r^{2}+y^{2}\right)}
$$

This is precisely the potential in five-dimensional flat space, where all the dimensions are large. From here we can also identify the five-dimensional gravitational constant $G_{5}=\left(3 \pi^{2} M^{3}\right)^{-1}[14]$.

\section{B. Four-dimensional point of view}

We now decompose the five-dimensional graviton $h_{M N}$ from the previous section into four-dimensional fields, and show that we obtain the same result for the gravitational potential using only four-dimensional fields and propagators. First the tensor structure is decomposed as

$$
h_{M N}=\left(\begin{array}{cc}
h_{\mu \nu} & V_{\mu} \\
V_{\nu} & S
\end{array}\right)
$$

When inserted into (3), we then obtain

$$
\begin{aligned}
S= & \int d^{4} x d y\left\{-V_{\alpha, \beta} V^{\alpha, \beta}+V_{\alpha, \beta} V^{\beta, \alpha}\right. \\
& -\frac{1}{2} h_{, \alpha} h^{, \alpha}+\frac{1}{2} h_{\alpha \beta, \nu} h^{\alpha \beta, \nu}+h^{\alpha \nu}{ }_{, \alpha} h_{, \nu}-h^{\alpha \nu}{ }_{, \alpha} h^{\beta}{ }_{\nu, \beta} \\
& \left.-\frac{1}{2}\left[h_{\alpha \beta}^{\prime} h^{\prime \alpha \beta}-\left(h^{\prime}\right)^{2}\right]+S_{, \alpha} h^{, \alpha}-h^{\alpha \beta}{ }_{, \beta} S_{, \alpha}\right\}, \quad(26
\end{aligned}
$$

where the prime means the derivative with respect to $y$. In particular, we note that all terms with $y$-derivatives 
of $V^{\mu}$ and $S$ have cancelled, which means that only the graviton $h_{\mu \nu}$ will acquire a mass in four dimensions.

This is related to the gauge freedom of the fivedimensional theory, where a possible gauge is to demand that both $V^{\mu}$ and $S$ are independent of $y$, as we will now show. Starting with the five-dimensional coordinate transformation

$$
x^{M} \rightarrow \bar{x}^{M}=x^{M}+2 M^{-3 / 2} \xi^{M},
$$

the five-dimensional graviton field is transformed as

$$
h_{M N} \rightarrow \bar{h}_{M N}=h_{M N}-\left(\partial_{M} \xi_{N}+\partial_{N} \xi_{M}\right) .
$$

Using (25), we then get for the different components

$$
\begin{aligned}
\bar{h}_{\mu \nu} & =h_{\mu \nu}-\left(\partial_{\mu} \xi_{\nu}+\partial_{\nu} \xi_{\mu}\right), \\
\bar{V}_{\mu} & =V_{\mu}-\partial_{\mu} \xi_{4}-\xi_{\mu}^{\prime}, \\
\bar{S} & =S-2 \xi_{4}^{\prime} .
\end{aligned}
$$

By choosing a suitable function $\xi_{4}$ we can simplify the radion $S$. It is not possible to gauge the field away since $\xi_{4}$ must be periodic under $y \rightarrow L+y$, but the choice

$$
\xi_{4}(x, y)=\frac{1}{2} \int_{0}^{y} S\left(x, y^{\prime}\right) d y^{\prime}-\frac{y}{2 L} \int_{0}^{L} S\left(x, y^{\prime}\right) d y^{\prime}+\bar{\xi}(x)
$$

with $\bar{\xi}(x)$ arbitrary, removes the $y$-dependence of $S$ :

$$
\bar{S}(x, y)=\frac{1}{L} \int_{0}^{L} S\left(x, y^{\prime}\right) d y^{\prime}=\bar{S}(x) .
$$

We also see that $\xi_{4}$ clearly satisfies the correct periodic boundary conditions. Hence, by a choice of coordinates for the extra dimension, the radion can be taken to be independent of $y$. This is actually quite natural, since the physical interpretation of the radion is that it measures the physical size of the torus - a quantity that necessarily depends only on the four-dimensional coordinate $x$.

Similarly, we choose $\xi_{\mu}$ to remove the $y$-dependence from the vector field $V_{\mu}$. Again, it is not possible to remove the vector field completely since $\xi_{\mu}$ must be periodic. With the notation $W_{\mu} \equiv V_{\mu}-\partial_{\mu} \xi_{4}$, we write

$\xi_{\mu}(x, y)=\int_{0}^{y} W_{\mu}\left(x, y^{\prime}\right) d y^{\prime}-\frac{y}{L} \int_{0}^{L} W_{\mu}\left(x, y^{\prime}\right) d y^{\prime}+\bar{\xi}_{\mu}(x)$,

with $\bar{\xi}_{\mu}(x)$ arbitrary. The transformed vector field is then

$$
\bar{V}_{\mu}(x, y)=\frac{1}{L} \int_{0}^{L} W_{\mu}\left(x, y^{\prime}\right) d y^{\prime}=\bar{V}_{\mu}(x) .
$$

A choice of four-dimensional coordinates can therefore make the vector field independent of $y$. We call the gauge where both $V_{\mu}$ and $S$ are independent of $y$ for the unitary gauge, and use it in the following. Note that the zero modes of both $V^{\mu}$ and $S$ are physical degrees of freedom with torus compactification. It is not possible to choose a gauge where either of them disappears completely.

The graviton field $h_{\mu \nu}$ is expanded in plane waves:

$$
h_{\mu \nu}(x, y)=\sum_{p} h_{\mu \nu}^{p}(x) \psi_{p}(y), \quad \psi_{p}(y)=\frac{1}{\sqrt{L}} e^{i p y} .
$$

In order to remove the crossterms between $h_{\mu \nu}$ and $S$ in (26), which only affects the zero mode $h_{\mu \nu}^{0}$ since $S$ is independent of $y$, we redefine $h_{\mu \nu}^{0}$ as

$$
h_{\mu \nu}^{0} \rightarrow h_{\mu \nu}^{0}+\frac{1}{\sqrt{6}} \eta_{\mu \nu} \phi,
$$

where we have also rescaled the scalar field as

$$
S=\sqrt{\frac{2}{3 L}} \phi,
$$

in order to get a canonical Lagrangian for $\phi$. If we also make a trivial rescaling $V^{\mu}=A^{\mu} / \sqrt{2 L}$ of the vector field, the action is reduced to

$$
\begin{aligned}
S=\int d^{4} x\left\{-\frac{1}{2} A_{\mu, \nu} A^{\mu, \nu}+\frac{1}{2} A_{\mu, \nu} A^{\nu, \mu}+\frac{1}{2} \phi_{, \mu} \phi^{, \mu}\right. \\
+\sum_{p}\left[-\frac{1}{2} h_{, \alpha}^{p} h_{-p}^{, \alpha}+\frac{1}{2} h_{\alpha \beta, \nu}^{p} h_{-p}^{\alpha \beta, \nu}+h_{p, \alpha}^{\alpha \nu} h_{, \nu}^{-p}\right. \\
\left.\left.\quad-h_{p, \alpha}^{\alpha \nu} h_{-p \nu, \beta}^{\beta}-\frac{1}{2} p^{2}\left(h_{\alpha \beta}^{p} h_{-p}^{\alpha \beta}-h^{p} h^{-p}\right)\right]\right\},
\end{aligned}
$$

after performing the $y$-integration. From this effective four-dimensional action we can clearly identify the physical fields as a massless graviton $(p=0)$, a tower of massive gravitons with Lagrangians identical to (12), a massless vector field, and a massless scalar field.

As we can see from (30) and (32), the unitary gauge is not uniquely determined - we can still make transformations in the form of the two functions $\bar{\xi}(x)$ and $\bar{\xi}_{\mu}(x)$ which only depend on the four-dimensional coordinate $x$. This corresponds to the transformations

$$
\begin{aligned}
h_{\mu \nu}^{0} & \rightarrow h_{\mu \nu}^{0}-\left(\partial_{\mu} \bar{\xi}_{\nu}+\partial_{\nu} \bar{\xi}_{\mu}\right), \\
A_{\mu} & \rightarrow A_{\mu}-\partial_{\mu} \bar{\xi},
\end{aligned}
$$

of the zero mode of the graviton and the vector field, and is precisely what allows us to identify these fields as massless graviton and vector fields in four dimensions. Also note that both actions (26) and (37) are invariant under this residual gauge transformation, meaning that the order which we perform the gauge transformation and the field redefinition (35) doesn't matter.

The interaction with matter in five dimensions can be written $S_{\text {int }}=-\int d^{4} x d y M^{-3 / 2} h_{M N} T^{M N}$. For a source located at $y=y^{\prime}$ the energy momentum tensor is

$$
T^{M N}(x, y)=\delta_{\mu}^{M} \delta_{\nu}^{N} T^{\mu \nu}(x) \delta\left(y-y^{\prime}\right) .
$$

Using the expansion (34) and the redefinition (35) we therefore get the effective four-dimensional interaction

$$
\mathcal{L}_{\mathrm{int}}=-\frac{M^{-3 / 2}}{\sqrt{L}}\left(\sum_{p} e^{i p y^{\prime}} h_{\mu \nu}^{p} T^{\mu \nu}+\frac{1}{\sqrt{6}} \phi T\right) .
$$


The radion field thus couples to the trace of the energy momentum tensor.

From (37) and (40) we can now obtain the gravitational potential in the effective four-dimensional theory:

$$
\begin{aligned}
V(\boldsymbol{k}, y) & =\sum_{h_{\mu \nu}^{0}}+\sum_{p \neq 0} h_{\mu \nu}^{p}+m_{1} m_{2} \\
& \left.=-\frac{1}{M^{3} L} \frac{1}{\boldsymbol{k}^{2}}+\frac{2}{3} \sum_{p \neq 0} \frac{e^{i p y}}{\boldsymbol{k}^{2}+p^{2}}+\frac{1}{6} \frac{1}{\boldsymbol{k}^{2}}\right] \\
& =-\frac{2 m_{1} m_{2}}{3 M^{3} L} \sum_{\text {all } p} \frac{e^{i p y}}{\boldsymbol{k}^{2}+p^{2}}
\end{aligned}
$$

The factors $1 / 2$ and $2 / 3$ for the gravitons originate from the polarization tensors (10) and (17) for $D=4$, whereas the factor $1 / 6$ for the radion follows from the interaction (40). Thus, we see that the massless graviton and the radion combined give exactly the same overall factor as the massive gravitons. By Fourier inverting (411), we get the same result as (21) derived in the previous section from the five-dimensional point of view.

\section{THE RANDALL-SUNDRUM MODEL WITH TWO BRANES}

In the fine-tuned or critical RS model, the background metric is given by

$$
\begin{aligned}
d s^{2} & =A^{2}(y) \eta_{\mu \nu} d x^{\mu} d x^{\nu}-d y^{2} \\
& =A^{2}(z)\left(\eta_{\mu \nu} d x^{\mu} d x^{\nu}-d z^{2}\right),
\end{aligned}
$$

where

$$
A(y)=e^{-\mu|y|}, \quad A(z)=\frac{1}{1+\mu|z|},
$$

is the warp factor in the physical coordinate $y$ and the conformal coordinate $z$, respectively. The visible brane is located at $y=z=0$ and the hidden brane at $y=y_{r}$, $z=z_{r}$. We assume the usual orbifold symmetry $S^{1} / Z_{2}$ for the fifth dimension, which basically means that the branes are located at the endpoints of this dimension. The visible and hidden brane have tension $\lambda=6 \mu M^{3}$ and $-\lambda$, respectively, and the cosmological constant in the anti de Sitter bulk space is $\Lambda_{B}=-6 \mu^{2}$. The finetuning between the positive tension on the visible brane and the negative cosmological constant in the bulk means that the effective four-dimensional cosmological constant vanishes, and the four-dimensional space is therefore flat with metric $\eta_{\mu \nu}$. For a more detailed discussion on the phenomenology of the RS model, see e.g. 10].

\section{A. Graviton Lagrangian}

Using the conformal $z$-coordinate, we write the perturbed metric as

$$
g_{M N}=A^{2}(z)\left(\eta_{M N}+h_{M N}\right) .
$$

The derivation of the Lagrangian for $h_{M N}$ is easiest to do using this coordinate, but still involves some rather tedious calculations (see appendix $\mathrm{A}$ ). The starting point is the action

$$
\begin{aligned}
S= & -\frac{1}{2} M^{3} \int d^{4} x d z \sqrt{\left|g_{M N}\right|}\left(R+2 \Lambda_{B}\right) \\
& -\lambda \int d^{4} x d z \sqrt{\left|g_{\mu \nu}\right|}\left[\delta(z)-\delta\left(z-z_{r}\right)\right] .
\end{aligned}
$$

Here $\sqrt{\left|g_{M N}\right|}$ means the square root of the determinant of the five-dimensional metric, i.e.

$$
\begin{aligned}
\sqrt{\left|g_{M N}\right|}=A^{5}[ & 1+\frac{1}{2}(h-S)+\frac{1}{8}(h-S)^{2} \\
& \left.-\frac{1}{4} h^{\mu \nu} h_{\mu \nu}+\frac{1}{2} V^{\mu} V_{\mu}-\frac{1}{4} S^{2}\right],
\end{aligned}
$$

where we have used (25) for $h_{M N}$, whereas $\sqrt{\left|g_{\mu \nu}\right|}$ only involves the four-dimensional components, i.e.

$$
\sqrt{\left|g_{\mu \nu}\right|}=A^{4}\left[1+\frac{1}{2} h+\frac{1}{8} h^{2}-\frac{1}{4} h^{\mu \nu} h_{\mu \nu}\right] .
$$

From the background solution (43) it follows that both the zeroth order and the first order parts of (45) vanish, as they should. For the second order part, the result A99 of appendix $\mathrm{A}$ then implies

$$
\begin{gathered}
S=-\frac{1}{2} M^{3} \int d^{4} x d y\left\{A^{2}\left[\frac{1}{2} V_{\alpha, \beta} V^{\alpha, \beta}-\frac{1}{2} V_{\alpha, \beta} V^{\beta, \alpha}\right]\right. \\
+A^{2}\left[-\frac{1}{4} h_{\alpha \beta, \nu} h^{\alpha \beta, \nu}-\frac{1}{2} h^{\alpha \beta}{ }_{, \beta} h_{, \alpha}+\frac{1}{2} h^{\alpha \nu}{ }_{\alpha} h^{\beta}{ }_{\nu, \beta}\right. \\
\left.\quad+\frac{1}{4} h_{, \alpha} h^{, \alpha}+\frac{1}{2} h^{\alpha \beta}{ }_{, \beta} S_{, \alpha}-\frac{1}{2} h_{, \alpha} S^{, \alpha}\right] \\
\left.+A^{4}\left[\frac{1}{4} h_{\alpha \beta}^{\prime} h^{\prime \alpha \beta}-\frac{1}{4}\left(h^{\prime}\right)^{2}\right]-\frac{3}{2} A^{3} A^{\prime} S h^{\prime}-3 \mu^{2} A^{4} S^{2}\right\},
\end{gathered}
$$

where we have also changed the integration variable to $y$, and the prime means derivative with respect to $y$. Again we note that all terms with $y$-derivatives of $V^{\mu}$ and $S$ have disappeared, so the graviton field will be the only massive field in four dimensions (the $S^{2}$-term will be removed by a field redefinition). However, in contrast to the torus, it is now possible to choose a gauge where the vector field disappears completely.

The issue of gauge transformations in the RS model has been discussed in detail by Boos et al. [9], but we include the main parts here for completeness. In the RS braneworld there is an additional symmetry that is not present for the torus, namely the orbifold symmetry $S^{1} / Z_{2}$. As we will see, this symmetry is what removes the vector field as a physical degree of freedom. This conclusion can actually be reached almost trivially by just 
looking at the orbifold symmetries that the components of the five-dimensional graviton must satisfy:

$$
\begin{aligned}
h_{\mu \nu}(x,-z) & =h_{\mu \nu}(x, z), \\
V_{\mu}(x,-z) & =-V_{\mu}(x, z), \\
S(x,-z) & =S(x, z) .
\end{aligned}
$$

We expect that the $z$-dependence of the vector field can be removed just as for the torus, but additionally, since $V_{\mu}(z)$ is an odd function of $z$, it cannot have a zero mode either. Thus, it should be possible to choose a gauge where the vector field disappears altogether.

To see this in more detail, we start with the fivedimensional coordinate transformation $x^{M} \rightarrow \bar{x}^{M}=$ $x^{M}+\xi^{M}$, which transforms the graviton as

$$
h_{M N} \rightarrow \bar{h}_{M N}=h_{M N}-A^{-2}\left(\nabla_{M} \xi_{N}+\nabla_{N} \xi_{M}\right),
$$

where the covariant derivative is with respect to the background metric $g_{M N}=A^{2}(z) \eta_{M N}$. Writing the gauge functions as

$$
\xi_{\mu}=A^{2} \hat{\xi}_{\mu}, \quad \xi_{4}=A \hat{\xi}_{4},
$$

for convenience, the components of $h_{M N}$ transform as

$$
\begin{aligned}
\bar{h}_{\mu \nu} & =h_{\mu \nu}-\left(\partial_{\mu} \hat{\xi}_{\nu}+\partial_{\nu} \hat{\xi}_{\mu}-\frac{2 A^{\prime}}{A^{2}} \eta_{\mu \nu} \hat{\xi}_{4}\right), \\
\bar{V}_{\mu} & =V_{\mu}-\left(\frac{1}{A} \partial_{\mu} \hat{\xi}_{4}+\hat{\xi}_{\mu}^{\prime}\right) \\
\bar{S} & =S-\frac{2}{A} \hat{\xi}_{4}^{\prime}
\end{aligned}
$$

where the prime means the derivative with respect to $z$. We choose the gauge where

$$
\hat{\xi}_{\mu}(x,-z)=\hat{\xi}_{\mu}(x, z), \quad \hat{\xi}_{4}(x,-z)=-\hat{\xi}_{4}(x, z) .
$$

The $z$-dependence of the radion is removed by choosing

$$
\hat{\xi}_{4}(x, z)=\frac{1}{2} \int_{0}^{z} A\left(z^{\prime}\right) S\left(x, z^{\prime}\right) d z^{\prime}-\frac{y}{2 y_{r}} \int_{0}^{z_{r}} A\left(z^{\prime}\right) S\left(x, z^{\prime}\right) d z^{\prime} .
$$

We then get, by using $d y / d z=A(z)$,

$$
\begin{aligned}
\bar{S}(x, z) & =\frac{1}{y_{r}} \int_{0}^{z_{r}} A\left(z^{\prime}\right) S\left(x, z^{\prime}\right) d z^{\prime} \\
& =\frac{1}{y_{r}} \int_{0}^{y_{r}} S\left(x, y^{\prime}\right) d y^{\prime}=\bar{S}(x) .
\end{aligned}
$$

Note that we can not add an arbitrary function $\bar{\xi}(x)$ in (54), in contrast to the torus, since this would break the orbifold symmetry of $\hat{\xi}_{4}$. This is another hint that the vector field must disappear, since we don't have any residual gauge transformations left that would transform the massless four-dimensional vector field. Also note that we impose the orbifold symmetry both before and after the coordinate transformation, which means that the visible brane remains straight in position $\bar{z}=0$. This is also clearly seen from the fact that $\hat{\xi}_{4}(z=0)=0$. Thus, we do not consider brane bending here, since brane bending can always be removed by a coordinate transformation [3] .

Moving on to the vector field, we see that we obtain $\bar{V}_{\mu}=0$ if we choose

$$
\hat{\xi}_{\mu}(x, z)=\int_{0}^{z} W_{\mu}\left(x, z^{\prime}\right) d z^{\prime}+\bar{\xi}_{\mu}(x),
$$

where $W_{\mu} \equiv V_{\mu}-A^{-1} \partial_{\mu} \hat{\xi}_{4}$, and the function $\bar{\xi}_{\mu}(x)$ can be chosen arbitrarily. The reason why this works in the RS model but not for the torus, is that the function $W_{\mu}$ is now an odd function of $z$, which implies both the correct orbifold symmetry $\hat{\xi}_{\mu}(-z)=\hat{\xi}_{\mu}(z)$, and the periodic boundary condition $\hat{\xi}_{\mu}\left(2 z_{r}+z\right)=\hat{\xi}_{\mu}(z)$. The latter follows from the identity $\int_{0}^{2 z_{r}} W_{\mu}(z) d z=\int_{-z_{r}}^{z_{r}} W_{\mu}(z) d z=0$. Thus, we see that the vector field is a pure gauge mode in the RS model, and by choosing the four-dimensional coordinates favourably we can make the field vanish. We call the gauge where $V_{\mu}=0$ and $S$ is independent of $z$ (or $y$ ) for the unitary gauge in the RS model, and use it in the following.

Next, we turn to the crossterms between $h_{\mu \nu}$ and $S$. Because they are a bit more involved than for the torus, we get the more complicated redefinition

$$
h_{\mu \nu} \rightarrow h_{\mu \nu}+f(y) \eta_{\mu \nu} S+g(y) \partial_{\mu} \partial_{\nu} S,
$$

where

$$
\begin{aligned}
& f(y)=\mu|y|+f_{0}, \quad f_{0}=\frac{\mu y_{r}}{e^{2 \mu y_{r}}-1}, \\
& g(y)=\frac{1}{4 \mu^{2}}\left\{f_{0}\left[e^{2 \mu|y|}-1\right]^{2}+e^{2 \mu|y|}[1-2 \mu|y|]-1\right\}+g_{0} .
\end{aligned}
$$

The constant $f_{0} \equiv f(0)$ is determined by requiring that the crossterms disappear on the branes, i.e. that we don't have any delta function crossterms. (More precisely, this implies the conditions $g^{\prime}\left(0^{+}\right)=g^{\prime}\left(y_{r}^{-}\right)=0$.) The constant $g_{0} \equiv g(0)$, however, doesn't seem to have any constraints, and can therefore be chosen freely. In any case, its value will not affect the gravitational potential.

With the field redefinition (57), all crossterms between $h_{\mu \nu}$ and $S$ and also the $S^{2}$ term disappear from (48), and we are left with a kinetic term $S_{, \alpha} S^{, \alpha}$ for the scalar field with a coefficient

$$
-\frac{1}{2} M^{3} \int_{-y_{r}}^{y_{r}} \frac{3}{2}\left(f^{2}-f\right) A^{2} d y=\frac{\frac{3}{4} M^{3} \mu y_{r}^{2}}{e^{2 \mu y_{r}}-1} .
$$

The physical radion $\phi$ with a kinetic term $\frac{1}{2} \phi_{, \alpha} \phi^{, \alpha}$ must therefore be given by

$$
\phi=\sqrt{\frac{\frac{3}{2} M^{3} \mu y_{r}^{2}}{e^{2 \mu y_{r}}-1}} S .
$$

In particular, we note that the radion drops out of the action in the limit $y_{r} \rightarrow \infty$ of one brane, as also 
shown in [9]. Finally we expand $h_{\mu \nu}$ in terms of fourdimensional eigenstates

$$
h_{\mu \nu}(x, y)=2 M^{-3 / 2} \sum_{m} h_{\mu \nu}^{m}(x) \Phi_{m}(y),
$$

where the functions $\Phi_{m}(y)$ satisfy the following wave equation and normalization:

$$
\begin{aligned}
\Phi_{m}^{\prime \prime}(y)+\frac{4 A^{\prime}}{A} \Phi_{m}^{\prime}(y)+\frac{m^{2}}{A^{2}} \Phi(y) & =0 \\
\int_{-y_{r}}^{y_{r}} A^{2}(y) \Phi_{m}(y) \Phi_{m^{\prime}}(y) d y & =\delta_{m, m^{\prime}}, \\
\int_{-y_{r}}^{y_{r}} A^{4}(y) \Phi_{m}^{\prime}(y) \Phi_{m^{\prime}}^{\prime}(y) d y & =m^{2} \delta_{m, m^{\prime}} .
\end{aligned}
$$

(The last of these equations follows trivially from the first two.) The general solution to (62) can be expressed in terms of Bessel functions, see e.g. 7] for more details. Combining all of this, we are finally able to reduce (48) to the effective four-dimensional action

$$
\begin{aligned}
S=\int d^{4} x\{ & \sum_{m}\left[-\frac{1}{2} h_{, \alpha}^{m} h_{m}^{, \alpha}+\frac{1}{2} h_{\alpha \beta, \nu}^{m} h_{m}^{\alpha \beta, \nu}+h_{m, \alpha}^{\alpha \nu} h_{, \nu}^{m}\right. \\
& \left.-h_{m, \alpha}^{\alpha \nu} h_{m \nu, \beta}^{\beta}-\frac{1}{2} m^{2}\left(h_{\alpha \beta}^{m} h_{m}^{\alpha \beta}-h_{m}^{2}\right)\right] \\
& \left.+\frac{1}{2} \phi_{, \mu} \phi^{, \mu}\right\} .
\end{aligned}
$$

Again we recognize this as a massless graviton $(m=0)$, a tower of massive gravitons, and a massless scalar field. The four-dimensional field content of the theory is therefore the same as with torus compactification, except for the vector field which is now absent.

From (54) and (56) we note that the only residual gauge symmetry we have left after imposing the unitary gauge, is a transformation of the four-dimensional coordinate by a function $\bar{\xi}_{\mu}(x)$ which only depends on $x$. The graviton field is then transformed as

$$
h_{\mu \nu}^{0} \rightarrow h_{\mu \nu}^{0}-\left(\partial_{\mu} \bar{\xi}_{\nu}+\partial_{\nu} \bar{\xi}_{\mu}\right) .
$$

This is precisely the transformation that allows us to identify the zero mode $h_{\mu \nu}^{0}$ of the five-dimensional graviton as a massless graviton in four dimensions. Again we also note that both actions (48) and (63) are invariant under this residual gauge transformation, so we can choose freely the order which we perform the field redefinition (57) and the gauge transformation.

\section{B. Interaction with matter}

With the source (39), the interaction is given by

$$
\begin{aligned}
S_{\mathrm{int}} & =\int d^{4} x d y \sqrt{g} \frac{1}{2} T_{M N} \delta g^{M N} \\
& =-\int d^{4} x\left[\frac{1}{2} A^{2}\left(y^{\prime}\right) T^{\mu \nu}(x) h_{\mu \nu}\left(x, y^{\prime}\right)\right] .
\end{aligned}
$$

From (57), (60) and (61), we have

$$
\begin{aligned}
& h_{\mu \nu}\left(x, y^{\prime}\right)=2 M^{-3 / 2} \sum_{m} h_{\mu \nu}^{m}(x) \Phi_{m}\left(y^{\prime}\right)+ \\
& \sqrt{\frac{e^{2 \mu y_{r}}-1}{\frac{3}{2} M^{3} \mu y_{r}^{2}}}\left[f\left(y^{\prime}\right) \eta_{\mu \nu}+g\left(y^{\prime}\right) \partial_{\mu} \partial_{\nu}\right] \phi(x) .
\end{aligned}
$$

Since the energy momentum tensor $T_{\mu \nu}$ is conserved, $\partial_{\mu} T^{\mu \nu}=0$, the term with $g\left(y^{\prime}\right)$ disappears after a partial integration, and we are left with

$$
\begin{aligned}
\mathcal{L}_{\text {int }} & =-M^{-3 / 2} A^{2}\left(y^{\prime}\right) \\
\times & \times\left[\sum_{m} \Phi_{m}\left(y^{\prime}\right) h_{\mu \nu}^{m} T^{\mu \nu}+f\left(y^{\prime}\right) \sqrt{\frac{e^{2 \mu y_{r}-1}}{6 \mu y_{r}^{2}}} \phi T\right] .
\end{aligned}
$$

Again we see that the radion couples to the trace of the energy momentum tensor.

\section{The gravitational potential}

With one source on the brane at $y=0$ and the other at the point $y$, the gravitational potential is

$$
\begin{aligned}
& V(\boldsymbol{k}, y)=+\sum_{m>0} h_{\mu \nu}^{0}-> \\
&=-\frac{m_{1} m_{2}}{M^{3} e^{2 \mu y}}\left\{\frac{\Phi_{0}(0) \Phi_{0}(y)}{2 \boldsymbol{k}^{2}}+\frac{2}{3} \sum_{m>0} \frac{\Phi_{m}(0) \Phi_{m}(y)}{\boldsymbol{k}^{2}+m^{2}}\right. \\
&\left.+\frac{e^{2 \mu y_{r}}-1}{6 \mu y_{r}^{2}} \frac{f(0) f(y)}{\boldsymbol{k}^{2}}\right\} .
\end{aligned}
$$

With $f(y)$ from (58) this implies

$$
\begin{aligned}
& V(r, y)=-\frac{m_{1} m_{2}}{8 \pi M^{3} e^{2 \mu y} r}\left\{\frac{\mu}{1-e^{-2 \mu y_{r}}}+\right. \\
& \left.\frac{4}{3} \sum_{m>0} \Phi_{m}(0) \Phi_{m}(y) e^{-m r}+\frac{1}{3}\left(\frac{\mu y}{y_{r}}+\frac{\mu e^{-2 \mu y_{r}}}{1-e^{-2 \mu y_{r}}}\right)\right\} .
\end{aligned}
$$

Here we have inserted the expression

$$
\Phi_{0}(y)=\sqrt{\frac{\mu}{1-e^{-2 \mu y_{r}}}}
$$

for the zero mode, which follows from (62). With both sources on the visible brane, i.e. by setting $y=0$, (69) is reduced to

$$
\begin{gathered}
V(r)=-\frac{m_{1} m_{2}}{8 \pi M^{3} r}\left\{\frac{\mu}{1-e^{-2 \mu y_{r}}}+\frac{4}{3} \sum_{m>0} \Phi_{m}^{2}(0) e^{-m r}\right. \\
\left.+\frac{1}{3} \frac{\mu e^{-2 \mu y_{r}}}{1-e^{-2 \mu y_{r}}}\right\} .
\end{gathered}
$$


Both the massless graviton and the radion contribute to the long range potential. The radion contribution is, however, suppressed by an extra factor $e^{-2 \mu y_{r}} \sim$ $\left(M_{\mathrm{EW}} / M_{\mathrm{Pl}}\right)^{2} \sim 10^{-30}$ if we interpret the RS1 model as a possible solution to the hierarchy problem. If we include the radion in the definition of the effective fourdimensional Planck mass, we get

$$
M_{\mathrm{Pl}}^{2}=\frac{M^{3}}{\mu} \frac{1-e^{-2 \mu y_{r}}}{1+\frac{1}{3} e^{-2 \mu y_{r}}},
$$

and (71) can then be written

$$
V(r)=-\frac{G m_{1} m_{2}}{r}\left\{1+\frac{4}{3 \mu} \frac{1-e^{-2 \mu y_{r}}}{1+\frac{1}{3} e^{-2 \mu y_{r}}} \sum_{m>0} \Phi_{m}^{2}(0) e^{-m r}\right\},
$$

where $G=\left(8 \pi M_{\mathrm{Pl}}^{2}\right)^{-1}$ is the gravitational constant. The whole effect of the radion is the factor $\left(1+\frac{1}{3} e^{-2 \mu y_{r}}\right)$ in the potential and in the four-dimensional Planck mass. The same factor also appears by the related calculation of similar effects in 5]. Again we see that the radion contribution disappears in the limit $y_{r} \rightarrow \infty$. In this limit, we can also show that [7]

$$
\lim _{y_{r} \rightarrow \infty} \Phi_{m}^{2}(0)=\frac{2 \mu}{\pi m z_{r}} \frac{1}{J_{1}^{2}\left(\frac{m}{\mu}\right)+Y_{1}^{2}\left(\frac{m}{\mu}\right)},
$$

whereas $\sum_{m} \rightarrow \int_{0}^{\infty} \frac{z_{r}}{\pi} d m$, with the result [4, 7]

$$
\begin{aligned}
& \lim _{y_{r} \rightarrow \infty} V(r)= \\
& \quad-\frac{G m_{1} m_{2}}{r}\left\{1+\frac{8}{3 \pi^{2}} \int_{0}^{\infty} \frac{e^{-m r}}{J_{1}^{2}\left(\frac{m}{\mu}\right)+Y_{1}^{2}\left(\frac{m}{\mu}\right)} \frac{d m}{m}\right\} .
\end{aligned}
$$

This expression can be expanded to high order in powers of $\mu r$ applicable to the limits $\mu r \gg 1$ and $\mu r \ll 1[$ ] $]$.

From (69) we also have the potential for the more general case when the two masses have different $y$ coordinates. For instance, one can consider one mass on the visible brane at $y=0$ and the other mass on the hidden brane at $y=y_{r}$. However, the interpretation of the resulting potential is not obvious. This question has recently been addressed in [15].

\section{SUMMARY}

In this paper we have derived the effective fourdimensional Lagrangian of gravity in two different higherdimensional theories, first using torus compactification and then in the Randall-Sundrum braneworld model, and we have used the result to calculate the Newtonian gravitational potential in the two models. In both cases we have seen that a radion - a massless scalar field - contributes to the potential. Since the radion is massless, this affects the long range gravitational force and therefore also the effective four-dimensional Planck mass.
The presence of the radion is a result of the finite size of the fifth dimension, and basically represents fluctuations in the circumference $L$ of the torus and in the brane separation $y_{r}$. In this paper we have simply ignored the question of stabilizing the extra dimension, and the radion is therefore massless. In a more realistic description, where the stabilization is achieved by a dynamical mechanism (see e.g. 16]), the radion usually becomes massive and will therefore not contribute to the long range gravitational force.

In our derivation for the torus, we have found that a massless vector field $A^{\mu}$ is part of the effective fourdimensional theory. This field does not contribute to the gravitational interaction when the source is at rest in the extra dimension. However, it still is a physical degree of freedom, and with a moving source it can potentially contribute. In the RS model, on the other hand, the additional orbifold symmetry effectively removes the vector field as a physical degree of freedom. It is then a pure gauge mode that is removed by a suitable choice of coordinates, which among other things requires that the visible brane remains unbent in position $y=0$. The hidden brane is still allowed to move, and the physical distance between the branes is precisely the radion field $\phi(x)$ which cannot be gauged away.

Acknowledgement: We want to thank David Langlois for useful discussions about the role of the scalar field in these kinds of models. This work has been supported by grant no. NFR 153577/432 and 159637/V30 from the Research Council of Norway.

\section{APPENDIX A: METRIC PERTURBATIONS IN A CONFORMALLY FLAT SPACE}

We consider an arbitrary conformally flat space with $D$ dimensions, where the perturbed metric can be written

$$
g_{\mu \nu}(x)=A^{2}(x)\left[\eta_{\mu \nu}+h_{\mu \nu}(x)\right] .
$$

Here the warp factor $A(x)$ is allowed to depend on all coordinates. The inverse metric is

$$
g^{\mu \nu}=A^{-2}\left[\eta^{\mu \nu}-h^{\mu \nu}+h^{\mu \sigma} h_{\sigma}{ }^{\nu}+\ldots\right] .
$$

The Lagrangian of the graviton field $h_{\mu \nu}(x)$ is found by expanding the action

$$
S=-\frac{1}{2} M^{D-2} \int d^{D} x \sqrt{g} R
$$

to the second order in $h_{\mu \nu}$. Of course, some other terms, like a cosmological constant, must also be included in order to get a non-trivial warp factor, but the curvature term is by far the hardest one to calculate. The determi- 
nant of the metric is expanded as

where $A_{\mu} \equiv \partial_{\mu} A$. The Ricci tensor is then found to be

$$
\begin{aligned}
\sqrt{g} & =A^{D} \sqrt{\operatorname{det}\left(\eta_{\mu \nu}+h_{\mu \nu}\right)} \\
& =A^{D} \cdot \exp \left[\frac{1}{2} \operatorname{Tr} \sum_{n=1}^{\infty} \frac{(-1)^{n+1}}{n}\left(h^{\mu}{ }_{\nu}\right)^{n}\right] \\
& =A^{D} \cdot \exp \left[\frac{1}{2} h-\frac{1}{4} h^{\alpha \beta} h_{\alpha \beta}+\ldots\right] \\
& =A^{D}\left[1+\frac{1}{2} h+\frac{1}{8} h^{2}-\frac{1}{4} h^{\alpha \beta} h_{\alpha \beta}+\ldots\right] .
\end{aligned}
$$

The Christoffel symbols are, to the second order,

$$
\begin{aligned}
& \Gamma_{\alpha \beta}^{\mu}=\frac{A_{\alpha}}{A} \delta_{\beta}^{\mu}+\frac{A_{\beta}}{A} \delta_{\alpha}^{\mu}-\frac{A^{\mu}}{A} \eta_{\alpha \beta} \\
& +\frac{A_{\nu}}{A} \eta_{\alpha \beta} h^{\mu \nu}-\frac{A^{\mu}}{A} h_{\alpha \beta}+\frac{1}{2}\left(h^{\mu}{ }_{\alpha, \beta}+h^{\mu}{ }_{\beta, \alpha}-h_{\alpha \beta}{ }^{, \mu}\right) \\
& +\frac{A_{\nu}}{A} h^{\mu \nu} h_{\alpha \beta}-\frac{A_{\nu}}{A} \eta_{\alpha \beta} h^{\mu \sigma} h_{\sigma}{ }^{\nu} \\
& -\frac{1}{2} h^{\mu \nu}\left(h_{\nu \alpha, \beta}+h_{\nu \beta, \alpha}-h_{\alpha \beta, \nu}\right),
\end{aligned}
$$

$$
\begin{aligned}
R_{\mu \nu}= & -(D-2) \frac{A_{\mu \nu}}{A}-\frac{A_{\alpha}^{\alpha}}{A} \eta_{\mu \nu}+(2 D-4) \frac{A_{\mu} A_{\nu}}{A^{2}}-(D-3) \frac{A^{\alpha} A_{\alpha}}{A^{2}} \eta_{\mu \nu} \\
& +\frac{A_{\sigma \alpha}}{A} \eta_{\mu \nu} h^{\sigma \alpha}-\frac{A_{\alpha}^{\alpha}}{A} h_{\mu \nu}+(D-3) \frac{A_{\sigma} A_{\alpha}}{A^{2}} \eta_{\mu \nu} h^{\sigma \alpha}-(D-3) \frac{A^{\alpha} A_{\alpha}}{A^{2}} h_{\mu \nu} \\
& +(D-2) \frac{A^{\sigma}}{2 A}\left(h_{\sigma \mu, \nu}+h_{\sigma \nu, \mu}-h_{\mu \nu, \sigma}\right)+\frac{1}{2}\left(h^{\alpha}{ }_{\mu, \nu \alpha}+h_{\nu, \mu \alpha}^{\alpha}-h_{, \mu \nu}-\square h_{\mu \nu}\right)+\frac{A^{\sigma}}{2 A} \eta_{\mu \nu}\left(2 h_{\sigma, \alpha}^{\alpha}-h_{, \sigma}\right) \\
& +\frac{A_{\sigma \alpha}}{A} h^{\sigma \alpha} h_{\mu \nu}-\frac{A_{\sigma \alpha}}{A} \eta_{\mu \nu} h^{\alpha \rho} h_{\rho}^{\sigma}+(D-3) \frac{A_{\sigma} A_{\alpha}}{A^{2}} h^{\sigma \alpha} h_{\mu \nu}-(D-3) \frac{A_{\sigma} A_{\alpha}}{A^{2}} \eta_{\mu \nu} h^{\alpha \rho} h_{\rho}^{\sigma} \\
& +\frac{A^{\rho}}{A} \eta_{\mu \nu} h^{\alpha \sigma}\left(\frac{1}{2} h_{\alpha \sigma, \rho}-h_{\rho \alpha, \sigma}\right)+\frac{A_{\sigma}}{A} \eta_{\mu \nu} h^{\rho \sigma}\left(\frac{1}{2} h_{, \rho}-h_{\rho, \alpha}^{\alpha}\right)+\frac{A^{\sigma}}{A} h_{\mu \nu}\left(h_{\sigma, \alpha}^{\alpha}-\frac{1}{2} h_{, \sigma}\right) \\
& -\frac{D-2}{2} \frac{A_{\sigma}}{A} h^{\rho \sigma}\left(h_{\rho \mu, \nu}+h_{\rho \nu, \mu}-h_{\mu \nu, \rho}\right)+\frac{1}{4}\left(h_{\sigma \mu, \nu}+h_{\sigma \nu, \mu}-h_{\mu \nu, \sigma}\right)\left(h^{, \sigma}-2 h_{, \alpha}^{\alpha \sigma}\right) \\
& \left.+\frac{1}{4} h_{\sigma \alpha, \mu} h^{\sigma \alpha}{ }_{, \nu}+\frac{1}{2} h_{\sigma \mu, \alpha} h_{\nu}{ }^{\sigma, \alpha}-\frac{1}{2} h_{\sigma \mu, \alpha} h_{\nu}^{\alpha, \sigma}+\frac{1}{2} h^{\alpha \sigma} h_{\mu \nu, \alpha \sigma}+h_{\alpha \sigma, \mu \nu}-h_{\alpha \mu, \nu \sigma}-h_{\alpha \nu, \mu \sigma}\right),
\end{aligned}
$$

and the curvature scalar is therefore

$$
\begin{aligned}
A^{2} R= & -2(D-1) \frac{A_{\alpha}^{\alpha}}{A}-(D-1)(D-4) \frac{A^{\alpha} A_{\alpha}}{A^{2}}+2(D-1) \frac{A_{\alpha \beta}}{A} h^{\alpha \beta} \\
& +(D-1)(D-4) \frac{A_{\alpha} A_{\beta}}{A^{2}} h^{\alpha \beta}+2(D-1) \frac{A_{\sigma}}{A} h^{\sigma \alpha}{ }_{, \alpha}-(D-1) \frac{A^{\sigma}}{A} h_{, \sigma}+h^{\alpha \beta}{ }_{, \alpha \beta}-\square h \\
& -2(D-1) \frac{A_{\alpha \beta}}{A} h^{\alpha \sigma} h_{\sigma}{ }^{\beta}-(D-1)(D-4) \frac{A_{\alpha} A_{\beta}}{A^{2}} h^{\alpha \sigma} h_{\sigma}{ }^{\beta}+(D-1) \frac{A^{\sigma}}{A} h^{\alpha \beta} h_{\alpha \beta, \sigma} \\
& -2(D-1) \frac{A^{\sigma}}{A} h^{\alpha \beta} h_{\sigma \alpha, \beta}-2(D-1) \frac{A_{\sigma}}{A} h^{\nu \sigma} h^{\alpha}{ }_{\nu, \alpha}+(D-1) \frac{A_{\sigma}}{A} h^{\nu \sigma} h_{, \nu} \\
& +\frac{3}{4} h_{\alpha \beta, \nu} h^{\alpha \beta, \nu}-\frac{1}{2} h_{\alpha \beta, \nu} h^{\nu \beta, \alpha}+h^{\alpha \beta}{ }_{, \beta} h_{, \alpha}-h_{, \alpha}^{\alpha \nu} h_{\nu, \beta}^{\beta}-\frac{1}{4} h_{, \alpha} h^{, \alpha}+h^{\alpha \beta} h_{, \alpha \beta}+h^{\alpha \beta} \square h_{\alpha \beta}-2 h_{\alpha}{ }^{\beta} h^{\alpha \nu}{ }_{, \nu \beta} .
\end{aligned}
$$


Combining (A4) and (A7) we get the rather complicated expression

$$
\begin{aligned}
A^{2-D} \sqrt{g} R= & -2(D-1) \frac{A_{\alpha}^{\alpha}}{A}-(D-1)(D-4) \frac{A^{\alpha} A_{\alpha}}{A^{2}}+2(D-1) \frac{A_{\alpha \beta}}{A} h^{\alpha \beta}+(D-1)(D-4) \frac{A_{\alpha} A_{\beta}}{A^{2}} h^{\alpha \beta} \\
& -(D-1) \frac{A_{\alpha}^{\alpha}}{A} h+2(D-1) \frac{A_{\sigma}}{A} h_{, \alpha}^{\sigma \alpha}-(D-1) \frac{A^{\sigma}}{A} h_{, \sigma}-\frac{1}{2}(D-1)(D-4) \frac{A^{\alpha} A_{\alpha}}{A^{2}} h^{\alpha \beta} h_{, \alpha \beta}^{\alpha \beta} \square h \\
& -2(D-1) \frac{A_{\alpha \beta}}{A} h^{\alpha \sigma} h_{\sigma}{ }^{\beta}-(D-1)(D-4) \frac{A_{\alpha} A_{\beta}}{A^{2}} h^{\alpha \sigma} h_{\sigma}{ }^{\beta}+(D-1) \frac{A^{\sigma}}{A} h^{\alpha \beta} h_{\alpha \beta, \sigma} \\
& -2(D-1) \frac{A^{\sigma}}{A} h^{\alpha \beta} h_{\sigma \alpha, \beta}-2(D-1) \frac{A_{\sigma}}{A} h^{\nu \sigma} h^{\alpha}{ }_{\nu, \alpha}+(D-1) \frac{A_{\sigma}}{A} h^{\nu \sigma} h_{, \nu}+(D-1) \frac{A_{\alpha \beta}}{A} h^{\alpha \beta} h \\
& +\frac{1}{2}(D-1)(D-4) \frac{A_{\alpha} A_{\beta}}{A^{2}} h^{\alpha \beta} h+(D-1) \frac{A_{\sigma}}{A} h_{, \alpha}^{\sigma \alpha} h_{,}-\frac{D-1}{2} \frac{A^{\sigma}}{A} h_{, \sigma} h-\frac{D-1}{4} \frac{A_{\alpha}^{\alpha}}{A} h^{2} \\
& -\frac{1}{8}(D-1)(D-4) \frac{A^{\alpha} A_{\alpha}}{A^{2}} h^{2}+\frac{D-1}{2} \frac{A_{\alpha}^{\alpha}}{A} h^{\mu \nu} h_{\mu \nu}+\frac{1}{4}(D-1)(D-4) \frac{A^{\alpha} A_{\alpha}}{A^{2}} h^{\mu \nu} h_{\mu \nu} \\
& +\frac{3}{4} h_{\alpha \beta, \nu} h^{\alpha \beta, \nu}-\frac{1}{2} h_{\alpha \beta, \nu} h^{\nu \beta, \alpha}+h^{\alpha \beta}{ }_{, \beta} h_{, \alpha}-h_{, \alpha}^{\alpha \nu} h_{\nu, \beta}^{\beta}-\frac{1}{4} h_{, \alpha} h^{, \alpha} \\
& +h^{\alpha \beta} h_{, \alpha \beta}+h^{\alpha \beta} \square h_{\alpha \beta}-2 h_{\alpha}{ }^{\beta} h^{\alpha \nu}{ }_{, \nu \beta}+\frac{1}{2} h^{\alpha \beta}{ }_{, \alpha \beta} h-\frac{1}{2} h \square h .
\end{aligned}
$$

When inserted into the integral of the action, the zeroth order and first order parts of this expression should always vanish when the warp factor $A(x)$ is a solution to the background field equations. The second order part can be simplified using partial integrations, with the result

$$
\begin{aligned}
& S=-\frac{1}{2} M^{D-2} \int d^{D} x\left\{A ^ { D - 4 } \left[(D-1)(D-2) A_{\alpha} A_{\beta} h^{\alpha \sigma} h_{\sigma}{ }^{\beta}-\frac{1}{4}(D-1)(D-2) A_{\sigma} A^{\sigma} h^{\alpha \beta} h_{\alpha \beta}\right.\right. \\
&\left.-\frac{1}{2}(D-1)(D-2) A_{\alpha} A_{\beta} h^{\alpha \beta} h+\frac{1}{8}(D-1)(D-2) A_{\alpha} A^{\alpha} h^{2}\right] \\
&+(D-2) A^{D-3}\left[\frac{1}{2} A^{\alpha} h_{\alpha \beta, \nu} h^{\nu \beta}-A_{\alpha} h^{\alpha \beta} h_{, \beta}-A^{\nu} h^{\alpha \beta} h_{\alpha \beta, \nu}\right. \\
&\left.\quad+\frac{3}{2} A_{\beta} h_{\alpha}{ }^{\beta} h^{\alpha \nu}{ }_{, \nu}-\frac{1}{2} A_{\beta} h^{\alpha \beta}{ }_{, \alpha} h+\frac{1}{2} A^{\alpha} h h_{, \alpha}\right] \\
&\left.+A^{D-2}\left[-\frac{1}{4} h_{\alpha \beta, \nu} h^{\alpha \beta, \nu}-\frac{1}{2} h^{\alpha \beta}{ }_{, \beta} h_{, \alpha}+\frac{1}{2} h^{\alpha \nu}{ }_{, \alpha} h^{\beta}{ }_{\nu, \beta}+\frac{1}{4} h_{, \alpha} h^{, \alpha}\right]\right\} .
\end{aligned}
$$

This result can then be applied to e.g. the RS model, provided we also include the cosmological constant and brane tension. With $A(x)=1$ we see that (A9) is trivially reduced to the flat space result (3).

[1] L. Randall and R. Sundrum, Phys. Rev. Lett. 83, 3370 (1999), hep-ph/9905221

[2] L. Randall and R. Sundrum, Phys. Rev. Lett. 83, 4690 (1999), hep-th/9906064

[3] J. Garriga and T. Tanaka, Phys. Rev. Lett. 84, 2778 (2000), hep-th/9911055 S.B. Giddings, E. Katz and L. Randall, J. High Energy Phys. 03, 023 (2000), hep-th/0002091 N. Deruelle and T. Doležel, Phys. Rev. D64, 103506 (2001), gr-qc/0105118

[4] D.J.H. Chung, L. Everett and H. Davoudiasl, Phys. Rev. D64, 065002 (2001), hep-ph/0010103

[5] M.N. Smolyakov and I.P. Volobuev, hep-th/0208025 (2002)

[6] C. Charmousis, R. Gregory and V.A. Rubakov, Phys. Rev. D62, 067505 (2000), hep-th/9912160 E. Kiritsis,
N. Tetradis and T.N. Tomaras, J. High Energy Phys. 03, 019 (2002), hep-th/0202037 S. Nojiri and S.D. Odintsov, Phys. Lett. B548, 215 (2002), hep-th/0209066 K. Ghoroku, A. Nakamura and M. Yahiro, Phys. Lett. B571, 223 (2003), hep-th/0303068

[7] P. Callin and F. Ravndal, Phys. Rev. D70, 104009 (2004), hep-ph/0403302

[8] P. Callin, hep-ph/0407054 (2004)

[9] E.E. Boos, Y.A. Kubyshin, M.N. Smolyakov and I.P. Volobuev, hep-th/0105304 (2001); Class. Quant. Grav. 19, 4591 (2002), hep-th/0202009

[10] P. Binétruy, C. Deffayet, U. Ellwanger and D. Langlois, Phys. Lett. B477, 285 (2000), hep-th/9910219 I. Brevik, K. Ghoroku, S.D. Odintsov and M. Yahiro, Phys. Rev. D66, 064016 (2002), hep-th/0204066 I. Brevik, K. 
Børkje and J.P. Morten, Gen. Rel. Grav. 36, 2021 (2004), gr-qc/0310103

[11] G.F. Giudice, R. Rattazzi and J.D. Wells, Nucl. Phys. B544, 3 (1999), hep-ph/9811291

[12] A. Kehagias and K. Sfetsos, Phys. Lett. B472, 39 (2000), hep-ph/9905417

[13] E.G. Floratos and G.K. Leontaris, Phys. Lett. B465, 95 (1999), hep-ph/9906238 H. Liu, hep-ph/0312200 (2003)

[14] In general, we define the gravitational constant $G_{D}$ in
$D$ spacetime dimensions so that the gravitational force is given by $F \equiv G_{D} m_{1} m_{2} / r^{D-2}=d V / d r$, meaning that $V(r)=-G_{D} m_{1} m_{2} /\left[(D-3) r^{D-3}\right]$.

[15] R. Arnowitt and J. Dent, Phys. Rev. D71, 124024 (2005), hep-th/0412016

[16] W.D. Goldberger and M.B. Wise, Phys. Rev. Lett. 83, 4922 (1999), hep-ph/9907447 Phys. Lett. B475, 275 (2000), hep-ph/9911457 